\title{
Schémas variationnels et gestion du non-standard en contextes français et maltais
}

\author{
Anne-Marie Bezzina \\ Faculté des Sciences de l'éducation, Université de Malte \\ anne-marie.bezzina@um.edu.mt
}

Résumé. Une visée comparative est adoptée dans cette étude traitant de la variation, notamment de la variation stylistique qui se produit en fonction du degré de formalité caractérisant la situation de communication et d'un nombre d'autres critères. La comparaison des situations sociolinguistiques présentes en contextes français et maltais permet de faire ressortir les caractéristiques propres à chaque situation. En français, la variation stylistique passe par le registre; en maltais, l'alternance codique maltais/anglais s'y ajoute pour la réalisation du style. Le français se distingue par une ampleur stylistique exceptionnelle, qui s'explique par diverses raisons culturelles, comme l'idéologie du standard et la portée de la littératie. Les deux cadres s'opposent par leurs processus de standardisation: le français, anciennement élu langue officielle, a naturellement suivi des schémas de progrès différents du maltais, langue officielle depuis bien moins longtemps, à l'héritage écrit qui ne remonte pas très loin dans le passé. Les deux langues partagent pourtant certains objectifs qui sous-tendent leurs efforts de protection. Des hypothèses sont émises quant à l'acceptabilité sociale de la variation aux différents niveaux d'analyse linguistique en maltais, et quant au type de variation proéminent dans cette langue, qui serait la variation sociale. Ces hypothèses sont construites par comparaison avec les arguments parallèles élaborés pour la situation française.

\begin{abstract}
This study adopts a comparative approach in dealing with language variation, mainly stylistic variation, which refers to the production of modulations in speech according to the degree of formality present in the communicative situation, and other criteria. A comparison of the sociolinguistic situations characterizing the French and Maltese contexts marks out the features defining each situation more clearly. In French, stylistic variation is realized through register; in Malta, Maltese/English code-switching is resorted to, alongside register, for style shifting. French stands out due to its wide range of style-sensitive features, a result of cultural realities, among which the heavy legacy of the written norm. In its standardization process, French, a centuries-old official language, naturally progressed differently from Maltese, which was much more recently elected as official language, and has a much younger literary heritage. Both languages nonetheless share some objectives in their self-protection efforts. Hypotheses are proposed as regards the degree of social acceptability of variation on different levels of linguistic analysis in Maltese, and as regards social variation being the dominant type of variation in this language. These hypotheses are constructed in comparison to parallel arguments previously advanced for the French situation by a number of sociolinguists.
\end{abstract}




\section{Définitions et orientations de l'étude}

La variation stylistique renvoie à la variation au sein de la production linguistique par des locuteurs individuels, par opposition à la variation sociale, qui se manifeste entre groupes de locuteurs en fonction de critères démographiques comme l'âge, le sexe, le cadre rural ou urbain et le niveau socio-économique (Gadet 2003). La variation stylistique se produit pour sa part selon les usages, en fonction des conditions de production linguistique et des différences entre oral et écrit. Une large gamme de facteurs conditionnent les changements stylistiques, dont la formalité de la situation, le ton (sérieux ou de plaisanterie), le chenal (écrit ou oral), mais aussi le contexte co-construit et reconstruit par les participants tout au long de l'interaction, à partir de leurs objectifs et des enjeux identitaires présents dans leurs interrelations (Coupland 2001, Schilling-Estes 2002, Gadet 2005). Cette étude traite de façon limitée de la variation géographique ou diatopique, davantage de la variation sociale ou diastratique, et notamment de la variation stylistique ou diaphasique. Ces phénomènes seront abordés dans le cadre d'une comparaison entre les situations linguistiques caractérisant le français de France et le maltais parlé à Malte.

Le français, langue anciennement standardisée, possédant une vieille tradition d'écriture, langue internationale, parlée par de nombreux millions d'habitants en France et dans bien d'autres contextes, est une langue largement étudiée sous de multiples aspects d'investigation linguistique. Il faut décrire en d'autres termes le maltais, langue standardisée depuis relativement peu, possédant une littérature foisonnante mais jeune, langue à usage presque exclusivement domestique, parlée par quelques centaines de milliers de Maltais habitants des îles maltaises (population 415,000) ou expatriés. La linguistique maltaise est une tout jeune discipline. Malte est un pays bilingue (maltais, anglais), alors que si l'on fait abstraction des langues régionales et du bilinguisme, par exemple français-arabe que l'on trouve répandu en banlieue, on peut décrire la France comme pays largement monolingue.

Pour diverses raisons, nous croyons utile et intéressant d'étudier chacune de ces deux langues selon la perspective de la variation, notamment stylistique, en suivant une approche comparative. L'apport d'une telle approche comparative nous semble recouvrir trois aspects différents. D'abord, les études réalisées sur la variation en français peuvent se prêter à l'analyse des phénomènes variationnels en d'autres langues moins étudiées, pour enrichir les connaissances qu'on en a. Deuxièmement, le travail comparatif réalisé par d'autres chercheurs en variation (Armstrong 2001 pour l'anglais et le français, Berruto (1987) et Gadet $(1998,2007)$ pour l'italien, l'anglais et le français) est une source d'inspiration pour s'interroger sue des questions concernant plusieurs autres langues, dans notre cas le maltais et le français. En troisième lieu, la description des réalités variationnelles de toute langue gagne à être comparée à celle d'une autre ; le contraste des données rend encore plus saillantes les spécificités propres à tout contexte.

Le contexte maltais étant peu connu, relativement à la situation linguistique en France, il est nécessaire d'ouvrir une parenthèse afin de décrire brièvement la situation sociolinguistique des îles maltaises. Malte fut colonie anglaise de 1800 jusqu'en 1964. De nos jours, le maltais est langue nationale et langue de l'Union Européenne ; le maltais et l'anglais sont les deux langues officielles de l'île. Le maltais est de loin la principale langue de communication orale, accompagné pourtant d'une alternance codique maltais/anglais très répandue. Dans les zones «branchées » de l'île, comme dans la ville de Sliema et les 
villes environnantes, une partie des habitants utilise l'anglais comme L1 ou pratique très fortement l'alternance codique. Il existe aussi dans cette région de l'île une variété du maltais, caractérisée par un accent affecté et souvent marquée par une forte alternance codique, que le reste de la population appelle par moquerie «tal-pepè » [des snobs]. Les trois cités historiques à l'est du Grand Port de la Valette, les zones rurales et l'île de Gozo ont leur(s) propre(s) dialecte(s) du maltais. Parmi les locuteurs d'anglais L1, et même parmi les locuteurs du maltais «standard », beaucoup de personnes voient d'un mauvais œil ces dialectes régionaux qu'ils considèrent comme «tal-hamalli » [des criards]; en revanche, l'emploi de l'anglais est très critiqué comme du snobisme par une bonne partie des locuteurs du maltais L1.

Le maltais est la langue de l'administration, du parlement, des tribunaux et de l'Eglise. Les émissions de télévision produites localement sont en maltais (les émissions et films importés étant passés en anglais). Pourtant, en dehors de la sphère des organes contrôlés ou influencés par l'Etat ou par l'Eglise, la formalité implique presque invariablement l'emploi de l'anglais, dans le secteur privé. La communication orale et écrite dans des contextes formels comme les colloques ou les assemblées générales annuelles des entreprises, et les échanges oraux entre certains experts comme le personnel médical à l'hôpital, ont lieu en anglais. L'anglais domine à l'écrit ; beaucoup de Maltais écrivent automatiquement en anglais, même pour des textos ou courriels informels. L'anglais est la langue de l'université, et le principal véhicule de l'enseignement dans les écoles. L'optimisme sur le progrès du maltais dans les contextes officiels est contrebalancé par le fait que l'anglais jouit d'un prestige plus marqué dans la mentalité générale. L'utilisation répandue de l'alternance codique prouve la croyance que recourir, même partiellement, à l'anglais, confère à l'individu un aura d'un statut social et d'un niveau d'éducation supérieurs.

\section{Types de variation présents dans les contextes français et maltais}

La première question traitée dans cette étude est si le français et le maltais se conforment à la théorie postulant une identité fonctionnelle entre la variation de registre en contexte monolingue et la variation réalisée à travers l'alternance codique en situation bilingue. L'une des premières recherches à avoir postulé cette identité des fonctions entre les deux types de variation est celle de Blom et Gumperz (1972), qui, en définissant l'alternance métaphorique à Hemnesberget, soutient que le choix entre le Ranamal (dialecte régional) et le Bokmal (norvégien standard) génère des implications qui ressemblent aux significations véhiculées par la fluctuation entre tyet $v y$ dans la littérature russe. Traugott et Romaine (1985), Bell (2001) et Gadet (2005) établissent un parallèle entre l'alternance codique en situation bilingue et le changement de style en situation monolingue : les motivations et les effets caractérisant l'alternance entre langues ne sont pas fondamentalement différents de ceux qui ont été identifiés comme alternances stylistiques à l'intérieur de la même langue.

Les réalités du contexte maltais nous amènent à penser que cette association de la variation passant par le style avec les sociétés monolingues et de la variation passant par l'alternance aux communautés bilingues est partiellement démentie par le cas de Malte. Il a été prouvé que les deux types de variation coexistent dans le cadre bilingue maltais : la variation stylistique y est certes réalisée à travers l'alternance codique anglais-maltais, l'anglais étant vu comme langue de prestige, mais la variation passant par le registre caractérise elle aussi indéniablement le maltais, comme il a été démontré à travers les implications sociolinguistiques du choix des conjonctions et adverbes, de la jonction propositionnelle et de la dislocation en cette langue (XX 201X). Le maltais, à la fois langue officielle et langue de la conversation quotidienne, se prête à une palette de variation de registre semblable à celle des langues des contextes monolingues.

Pour ce qui est du français de France, il est vrai que les locuteurs recourent à des formes associées à divers degrés de formalité selon les cadres participatif et spatio-temporel de la situation de communication. Gadet (2007) rend compte, dans les productions linguistiques d'une jeune locutrice, des formes qui distinguent le discours formel (1. conversation téléphonique avec un professeur de cours par correspondance) et tout à fait familier (2. dispute au téléphone avec le compagnon), qui représentent les deux points extrêmes du répertoire stylistique à l'oral de cette locutrice qui a consenti à se faire enregistrer, sans qu'elle sache quand cela se passait. Nous en reproduisons ici deux très brefs extraits : 


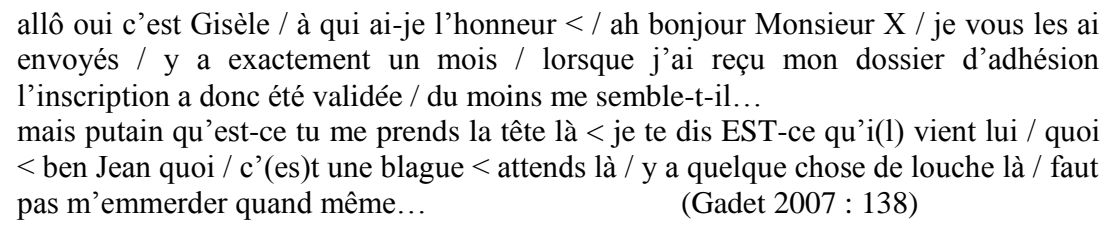

Parmi d'autres évidences de variation diaphasique en français, Coveney (1996) identifie la particule ne comme élément qui a perdu sa valeur de marqueur de variation sociale au profit d'une valeur indiquant la variation stylistique ; elle ne fait plus partie du système vernaculaire transmis de génération en génération par les processus d'acquisition normaux, mais est apprise plus tard dans la vie sous la pression de la langue écrite. Il trouve de l'évidence que les locuteurs modifient sensiblement le taux d'emploi de la particule selon le degré de formalité de la situation : un locuteur passe de $50 \%$ de rétention de nedans un premier entretien qui a lieu dans un cadre plus protocolaire, à $11,4 \%$ de rétention dans un second entretien plus décontracté, aux sujets qui tiennent de l'anecdotal.

On peut aussi mentionner le lexique variable qui caractérise le corpus de Dieuze recueilli par Armstrong (2001) à différents points de l'axe de la variation intralocuteur. Dans de tels cas il s'agit bien de variation stylistique, et non de variation sociale, car même les locuteurs français les plus éduqués possèdent les registres informels (Sanders 1993, George 1993).

Le français se conforme donc à la description de la variation passant par le registre. Le fait que des deux types de variation, il n'y en a qu'un de proéminent en France ne doit pas pour autant faire croire que la variation y soit peu importante : les chercheurs en variation estiment par contre que le français est une langue extrême en ce sens.

\section{L'ampleur de la variation en français}

Les chercheurs avancent deux arguments pour soutenir que l'éventail de la variation situationnelle soit plus large en français que dans d'autres langues : l'écart entre les formes écrites et orales, comme entre traits formels et informels, et deuxièmement, le fonctionnement particulier du lexique français. Lodge (1993) énumère plusieurs points linguistiques comme évidence que les différences syntaxiques entre les styles formel et informel se dégagent d'une façon particulièrement forte en français : les cas des systèmes temporels présents en français formel mais qui ont totalement disparu dans les variétés informelles, la négation en deux particules devenue rare en français informel, et l'inversion verbe-sujet si commune en style formel mais évitée dans un style informel. Quant au second argument, Lodge souligne que le système lexical du français a permis le développement d'un vocabulaire parallèle pour les objets communs, avec un grand nombre d'items qui existent en paires, l'un utilisé en variété formelle et l'autre en variété informelle.

\subsection{Raisons qui expliquent la configuration stylistique particulière du français}

\subsubsection{L'idéologie du standard : entre norme et purisme}

On peut paradoxalement retracer la cause de cette variabilité marquée du français dans les visées normatives, le purisme, la prescription et la vénération du standard inculqués dans la mentalité française par divers moyens. Pour Lodge (1993), les normes oppressives constituent toujours une incitation à la rebellion.

La croyance que l'hétérogénéité nuit à la communication efficace étant solidement ancrée dans la mentalité française, il en est résulté une forme standard rigoureusement codifiée qui exerce des pressions très lourdes sur les locuteurs (Ibid.). Dans des sociétés centralisées, urbanisées et socialement stratifiées comme celle de la France, il existe une forte pression institutionnelle pour se conformer aux normes sociales du comportement linguistique. Cette idéologie fondée sur le statut, liée au prestige apparent, est contrebalancée par les besoins des individus de former leur identité dans de plus petits groupes au sein de 
la société. Lodge constate que les locuteurs tendent à utiliser une langue non standard comme marque de leur adhésion au groupe et de solidarité entre ses membres. Le prestige latent au sein du groupe dépendra de l'habileté du locuteur à suivre les normes de sa communauté, qu'elle soit basée sur la classe, la région, l'ethnie ou l'âge. Il est possible qu'une idéologie du standard particulièrement puissante entraîne une plus grande pression sur les groupes minoritaires à se protéger et à resserrer les normes du comportement linguistique au sein de leur communauté (Ibid.).

\subsubsection{Une culture de littératie}

La seconde raison qui explique la richesse diaphasique particulière du français est le fait que cette langue survalorise l'écrit et «se situe à un pôle extrême parmi les cultures de littératie » (Gadet 2007: 58). Comme l'explique Lodge (1993), la tendance dans les cercles normatifs et toutes les institutions qui y puisent leur inspiration est de considérer la langue écrite comme le modèle privilégié du discours, supérieur à l'oral, car plus stable que lui. Ceci crée inévitablement de la tension avec les besoins du discours interactif quotidien (Ibid.).

\subsubsection{Le français : une langue particulièrement soumise à la standardisation et au nivellement}

Le prescriptivisme qui s'est traduit en processus de promotion d'une seule variété linguistique en France est l'un des facteurs responsables de la relative uniformisation de la phonologie en France. Les notions de standardisation et de nivellement se prêtent en effet à la description de la situation française. La standardisation est définie par Armstrong (2001) comme le processus de suppression de la variation linguistique suite à des initiatives institutionnelles, imposées sur la société, le plus souvent à travers la pression exercée par le système éducatif, et à travers les exemples de la presse. Le nivellement concerne la réduction des différences linguistiques entre variétés de langue, suite à des actes d'accommodation qui ont lieu de manière horizontale plutôt que hiérarchique, grâce à la mobilité sociale et géographique (Ibid.). A l'exclusion de la division nord-sud, le manque de variabilité régionale au niveau phonétique illustre le degré de standardisation atteint en français. Pour Armstrong, ceci distingue le français du cas de l'anglais britannique, par exemple, où chaque zone a son accent distinctif.

\subsubsection{Les influences culturelles}

Les raisons historiques qui expliquent le succès de la standardisation en français sont complexes. L'interférence de l'Etat en est partiellement responsable. Depuis le dix-huitième siècle, l'idée des EtatsNations se base sur le précepte d'une langue par état (Gadet 2007). Pour Armstrong (2001), la tradition française d'une forte gouvernance centralisée, impliquant la suppression impitoyable de centres de pouvoir régionaux, faisait partie d'une politique centralisatrice qui se reflétait dans les efforts pour codifier la langue standard écrite. La Révolution entraîna la volonté d'imposer la centralisation sur tous les citoyens, y compris la population rurale. L'aspect linguistique de cette entreprise était exprimé dans la devise 'La langue doit être une comme le peuple'. La langue française en vint donc à être identifiée avec l'identité nationale, et les langues régionales, avec un manque de loyauté vers la République. L'imposition d'une politique linguistique basée sur ces orientations idéologiques à travers l'éducation ne fut pourtant possible qu'à partir de la dernière partie du dix-neuvième siècle ; c'est donc la domination de Paris dans toutes les sphères de la vie sociale, même provinciale, qui assura la propagation de la langue standard avant l'école.

Les normes sociales évoquées par la langue standard sont selon Lodge (1993) renforcées par le rôle central que la langue a joué durant les deux siècles passés au niveau de la définition de l'identité nationale : la langue française est un symbole puissant qui fait naître chez les Français un sentiment de solidarité et de cohésion interne, en même temps qu'un sentiment de leur unicité face à d'autres nations, donc de distinctions externes. On peut noter un parallélisme sur ce plan avec les données culturelles de la situation maltaise, car la langue maltaise, tout comme, jusque dans un passé récent, le sentiment de 
cohésion nationale (cette fois face aux dominations étrangères), constituent deux des piliers qui définissent traditionnellement l'identité maltaise ${ }^{1}$ (Ebejer 1989, Cassar 2001, Borg et Mifsud 2005).

\subsection{Facteurs inhibant le maintien du standard : contextes favorisant la variation sociale, régionale et situationnelle}

Des facteurs constituant des obstacles à la propagation du standard font pourtant contrepoids aux facteurs consolidant sa dominance. Le Tableau 1 résume les points essentiels de la réflexion de Lodge (1993) sur les raisons d'existence des variétés dépourvues de prestige, qui s'opposent aux facteurs qui opèrent pour le maintien et la diffusion ultérieure du standard.

\begin{tabular}{|c|c|c|}
\hline & $\begin{array}{l}\text { Maintien } \\
\text { (du standard) }\end{array}$ & $\begin{array}{l}\text { Inhibition } \\
\text { (du standard) }\end{array}$ \\
\hline \multirow{3}{*}{ Société } & normes sociales & normes de communauté \\
\hline & & $\leftrightarrow$ \\
\hline & pressions du statut & pressions de solidarité \\
\hline \multirow{4}{*}{ Langue } & écrite & orale \\
\hline & formelle & informelle \\
\hline & publique & privée \\
\hline & discours planifié & discours non planifié \\
\hline
\end{tabular}

TABLEAU 1 : Facteurs de maintien et d'inhibition du standard (emprunté à Lodge 1993 : 234 ; notre traduction)

Les variétés linguistiques déterminées par la différence sociale se manifestent sous l'influence de l'âge et du sexe, ou émanent de la distance sociale selon le groupe géographique ou ethnique, ou encore la classe sociale. Si la constatation que les deux sexes parlent différemment doit être atténuée pour le cas de la France (Lodge 1993), les jeunes locuteurs s'éloignent de manière significative des normes à prestige, notamment au niveau du lexique. La langue des jeunes est délibérément non standard et contestataire (George 1993). La stratification sociale en province, qui se manifestait jadis par l'opposition entre les patois parlés par la population rurale et l'usage de type parisien des classes supérieures, a cédé la place à une variation diastratique (à base de classes sociales) selon le schéma parisien, suite à la 'mort' des patois et au développement des grandes conurbations en province (Lodge 1993). Le dernier facteur inhibant la progression du standard est la variation stylistique, qui nous occupera davantage ci-dessous par sa portée parmi les schémas variationnels en France.

\subsection{Considérations de l'amplitude variationnelle en maltais}

Comment juger quantitativement de la variation en maltais vis-à-vis de la variation si importante en français? Il est difficile de se prononcer alors qu'on en est encore aux toutes premières études touchant la variation stylistique et sociale en maltais. Les doublets lexicaux dont dispose le français pour de nombreux concepts communs contribuent majoritairement à sa richesse variationnelle. Où faut-il situer le maltais par rapport à ce phénomène ?

Le maltais s'est forgé à partir de sources multiples. Il est une langue à strates, chaque strate étant un héritage d'une domination étrangère ayant laissé des marques linguistiques ${ }^{2}$. Par conséquent, il possède lui aussi une grande quantité de doublets lexicaux pour de nombreux concepts, par exemple des paires de termes dont l'un dérive d'une source sémitique et l'autre d'une source romane ou anglo-saxonne, comme twegiba/risposta [réponse], tkellem/parla [il a parlé], jigifieri/voldieri [c'est-à-dire], jew/ossija [ou]. Alors qu'en français les doublets sont sensibles au registre, de telle façon qu'un terme non standard comme baraque, bagnole et fric n'est point utilisable dans des situations formelles, où s'imposent maison, voiture et argent, les restrictions d'emploi des doublets maltais ne sont pas du même genre. Il est possible que l'un des termes soit empreint de connotations négatives, comme paroli [balivernes] vis-à-vis de 
kliem[mots, propos], mais on peut envisager que paroli soit utilisé dans des cadres formels, si l'intention est de véhiculer précisément la valeur négative dont le terme est chargé. Dans certains doublets comme twegiba/risposta, jew/ossija, jigifieri/voldieri, les deux termes font partie du maltais standard. Il faudrait une étude beaucoup plus approfondie pour pouvoir trancher sur la valeur stylistique réelle de ces doublets en maltais.

Notre intuition est que si le maltais ne dispose peut-être pas en son sein d'une variation stylistique aussi saillante que celle du français, la possibilité du recours à l'alternance codique et à une prononciation régionale et/ou de classe sociale (vernaculaire ou affectée), fait ainsi que les locuteurs maltais disposent eux aussi d'une gamme variationnelle très large, par laquelle ils arrivent à se construire une identité particulière, et à adapter leurs réalisations linguistiques aux diverses situations de communication. Une considération du processus de standardisation du maltais révélera les points variationnels mis en valeur dans l'esprit maltais.

\section{Orientations majeures du processus de standardisation en maltais}

Relativement au français, le maltais a assez récemment trouvé sa place de langue officielle et langue nationale. La langue demeura pendant bien des siècles virtuellement restreinte à l'oral ; la documentation écrite se faisait en latin ou dans les langues romanes, notamment en italien, qui était la langue des couches sociales dominantes, et plus tard en anglais, durant la période coloniale. Le processus de standardisation fut donc entamé relativement tard dans l'histoire de la langue, pour des motivations politiques : Caruana (2011) décrit l'effort pour améliorer le statut du maltais comme une quête patriotique.

Le processus de standardisation connut ses origines avec la fondation en 1920 de l-Ghaqda tal-Kittieba tal-Malti [l'Association des Ecrivains maltais], dont l'un des objectifs était celui de standardiser l'écriture du maltais. En 1924 l'Association publia ses recommandations pour un alphabet basé sur des critères phonétiques. En 1984, un addendum officiel fut ajouté à ce premier document, rendant compte des changements subis par le maltais au fil des ans. Ces modifications inclurent un document sur l'orthographe des néologismes anglo-saxons et romans. Ceci marque une évolution importante par rapport au document de 1924, d'orientation puriste, privilégiant les sources arabes de la langue.

En 1984, il y avait la conscience que la strate sémitique était devenue non productive, et que le changement linguistique était en manière générale dû à l'emprunt de termes d'origine anglo-saxonne et italienne. L'esprit des responsables de la standardisation du maltais s'ouvrait donc enfin sur cette question à propos d'une réalité pluri-centenaire par la constatation (très tardive) que les emprunts s'assimilaient à l'orthographe du maltais et par la reconnaissance qu'en écrivant le maltais, les utilisateurs, à part quelques militants (souvent écrivains) du maltais sémitique, ne se soucient pas de l'origine sémitique ou autre des termes Caruana signale l'orientation de la politique linguistique touchant le maltais vis-à-vis de la constitution en strates successives de la langue, de façon à ce que les strates plus récentes aient été surtout dans le passé considérées avec méfiance et mépris face à la pureté des fondements sémitiques de la langue (« languagepolicy in Malta wasdetermined in relation to the internal structures of the language. » (Caruana 2011).

Ceci ne signifie pas que les responsables de la standardisation aient adopté une attitude d'acceptation complète vis-à-vis des termes d'origine romane ou anglo-saxonne. La strate sémitique continue d'être privilégiée. Cela se voit dans les choix terminologiques imposés pour diverses sphères d'activité. L'organe qui est de nos jours officiellement responsable de l'avancement du maltais est le KunsillNazzjonalital-IlsienMalti [Conseil National de la langue maltaise] et non le KunsillNazzjonalitalLingwaMaltija(Ilsien étant le terme sémitique, Lingwa le terme roman pour langue). Les exercices scolaires qui pour les générations précédentes étaient la Komprensjoni [Compréhension] et la

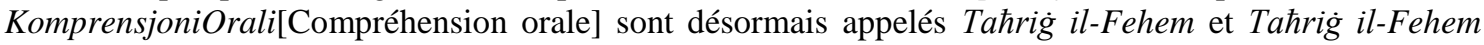
mis-Smigh. Ce genre de termes sémitiques recherchés peuvent évoquer chez le locuteur non spécialiste l'impression de contorsions artificielles, qu'on s'évertue pour chercher un terme «maltais » selon une formule où «maltais » égale «sémitique ». Cette idéologie semble par ailleurs avoir beaucoup pénétré l'esprit des utilisateurs, qui, s'ils n'utilisent pas forcément eux-mêmes les formes sémitiques au détriment 
des formes d'origine romane ou anglo-saxonne, continuent de les valoriser et de croire à leur supériorité (en termes de légitimité, de pureté, d'esthétique...). Une enquête sur les représentations linguistiques des Maltais (XXX 201X) revèle des réponses massivement approbatrices quant à l'emploi de l'expression $l$ ghodwa t-tajba plutôt quebong $u$ [litt. bon matin, d'origine sémitique et française respectivement, celle-là étant très rare et recherchée, et bongu le terme d'usage général].

Le vingt-et-unième siècle apporta l'inclusion de l-Att tal-Ilsien Malti [Acte de la langue maltaise] dans le code des lois maltaises et la création en 2005 du Kunsill Nazzjonali tal-Ilsien Malti [Conseil national de la langue maltaise], qui compte parmi ses missions principales celle de formuler une politique linguistique nationale, et celle de déterminer les règles qui doivent être adoptées à échelle nationale au niveau de l'orthographe maltaise. Malgré certaines contributions en matière de politique linguistique, comme la régulation du choix et de la qualité du maltais dans les médias (Rapport de 2009), et des exercices de consultation sur la distribution des langues dans l'éducation, la question de l'orthographe s'est à ce jour vu accorder beaucoup plus d'attention que la politique linguistique, bien plus délicate à cause de l'inclination d'une partie de la population vers l'anglais comme L1.

Sauf dans les écoles indépendantes (10\% de la population scolaire) où l'anglais est généralisé, l'anglais est véhicule de l'enseignement pour davantage de matières que le maltais dans les écoles publiques $(50 \%$ des enfants) et confessionnelles (40\% des enfants). Le curriculum national publié par le Département de l'Education en 1999 recommanda la séparation des langues dans les classes. Les documents subséquents n'ont plus reproduit ce genre d'intolérance vers l'alternance codique dans l'interaction verbale dans les cours. Camilleri Grima (2013) et Caruana (2011)plaident pour que l'on cesse de minimiser, voire de condamner le rôle de l'alternance codique dans le système scolaire, étant donné son utilité attestée dans les explications, et les bénéfices que les apprenants en tirent.

Caruana considère les nombreux emprunts utilisés aujourd'hui en maltais comme un signe de sa vitalité et de son dynamisme, et rend compte du fait qu'en réalité le maltais se distancie de ses origines sémitiques, et par le recours à de nombreux mots d'origine notamment anglo-saxonne, et par les schémas d'emploi du maltais écrit informel. A partir d'exemples de clavardage, XXX (201X) et Caruana (2011) constatent que l'orthographe de la communication technologique se base souvent sur des critères purement phonétiques. En adoptant ce genre d'écriture, les utilisateurs délaissent l'élément étymologique de l'orthographe standard du maltais, dédaignant ainsi le processus laborieux que la langue a dû subir pour atteindre sa forme standard (Caruana 2011).

Face à ces réalités, Caruana préconise l'honnêteté comme le principe qui devrait définir toute politique future de standardisation et d'emploi linguistique :

[...] the task of Maltese policy makers is not an easy one: on the one hand they have to 'safeguard' the largely unproductive Arabic stratum of the language and on the other hand they are to adopt measures to integrate innovations. In this vein, it may therefore be useful to recall the saying that 'honesty is the best policy': the more language policy takes authentic language use into consideration, the more it becomes legitimate and valid. (Caruana 2011)

\subsection{Parallélismes entre les processus de standardisation du maltais et du français}

La standardisation du maltais et du français diverge par l'aspect temporel. Dans l'histoire du français, le processus de suppression de la variation était certes sous la responsabilité d'organes officiels ou autorisés, mais il a aussi été accepté et «promulgué » par la population. Le nivellement qui se propageait au fil des siècles sous l'influence de Paris dans toutes les sphères de la vie sociale avait lieu de façon naturelle. Quant au maltais, si l'élection du dialecte de La Valette et de ses faubourgs comme variété standard se fit de manière naturelle de par l'importance administrative et commerciale de la capitale, donnant lieu à un certain nivellement phonologique, le processus de standardisation de l'écrit est assez jeune et imposé par les organes investis de l'autorité et les institutions accréditées. Il s'agit d'un processus mis en marche institutionnellement et délibérément accéléré, pour rattraper le retard des principes qui doivent gérer 
l'orthographe et la terminologie, et aussi pour faire face aux changements rapides que la langue est en train de subir.

La prescription accompagnant la standardisation dans les deux situations est un autre élément à propos duquel nous tenterons d'établir un rapprochement, en nous focalisant sur la variation régionale. Il semble que dans les deux pays, une période de reconnaissance et de valorisation officielles de ce type de variation soit actuellement en vigueur, suivant une période de mépris, voire de campagnes d'éradication, notamment dans le cas de la France. L'un des objectifs de l'école obligatoire mise en place au $19^{\mathrm{e}}$ siècle était l'éradication des patois. La croyance qu'ils empêchent l'apprentissage du français et de la culture mena à une véritable répression de ces parlers, et la forte centralisation linguistique qui rayonnait depuis Paris continua d'amoindrir leur portée. Ces méthodes et attitudes eurent un effet désastreux sur la situation des patois, comme sur l'image qu'avaient les locuteurs de leur langue et d'eux-mêmes.

De nos jours, les groupes de pression représentent les langues régionales de manière énergique, de sorte qu'elles ont trouvé leur place dans le système éducatif. Les régions les plus concernées ont mis en place une politique ambitieuse pour leur promotion, et une disposition a été introduite dans la Constitution à l'occasion de la réforme constitutionnelle du 21 juillet 2008 : au titre de l'article 75-1, les langues régionales appartiennent désormais « au patrimoine de la France ». Pourtant, ces termes semblent peindre les langues régionales comme quelque chose de tourné vers le passé.

On remarque la ressemblance avec les facteurs d'homogénéisation à l'œuvre dans le contexte maltais, seulement dans ce contexte-ci l'atténuation de la variété régionale a commencé à se produire plus tard, sans doute à cause de l'introduction plus tardive de l'école obligatoire. On note actuellement dans le cas de Malte un regain de l'intérêt pour les dialectes régionaux, symbolisé par la création de l'ONG VersAghtini il-Kelma Maltija [Donne-moi un vers Le mot maltais] avec l'objectif de les promouvoir. Cette nouvelle valorisation des dialectes va pourtant à l'encontre du mépris avec lequel ils sont traités par la plupart de la population, et, dans la vaste majorité des cas,les dialectophones adoptent tout de suite le maltais standard en parlant à des inconnus.

Il semble que dans les deux contextes la valorisation de la variété régionale se limite en fait à quelques cercles: des intellectuels et aimants du patrimoine, des hommes politiques qui y voient un enjeu particulier, et les utilisateurs dialectophones eux-mêmes, dont bon nombre ont pourtant intégré les attitudes de péjoration dirigées vers l'emploi des variétés régionales.

Quant aux objectifs qui sous-tendent la standardisation du français, ils répondent premièrement à la volonté de préserver son statut de langue internationale face à l'avancée d'autres langues dans ce domaine. Le deuxième objectif s'inspire du souci de protéger l'intégrité du français face aux emprunts introduits par les locuteurs, notamment à partir de l'anglais, et le troisième but est celui de sauvegarder la langue contre le mauvais usage de la part de ses utilisateurs mêmes. Le premier objectif n'est naturellement pas partagé par la standardisation du maltais, langue plus ou moins confinée à son pays d'origine, mais les deux autres constituent des objectifs que l'on avance comme justifications du processus pour le maltais également : il faut le protéger contre l'incursion et l'emploi exagéré de termes et de tournures anglais qui seraient en train de le déformer - la langue serait en danger. Gadet énumère les métaphores des menaces utilisées pour le cadre français «sur les registres du patrimoine en péril, de la guerre, de la contamination ou du viol », «l'ennemi d'aujourd'hui » étant l'anglais (Gadet 2007: 31). Dans le contexte maltais, les soucis qui instiguent certaines mesures, comme l'évitement de l'anglais aux médias, sont identiques: l'adoption dans les deux langues de nombreux mots et expressions anglais risque de leur faire perdre leur cohérence et l'harmonie interne (Judge 1993, pour le français). Les métaphores de la menace et de la honte foisonnent à propos du maltais chez des personnages importants

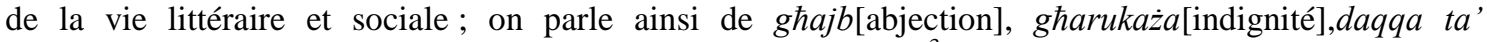

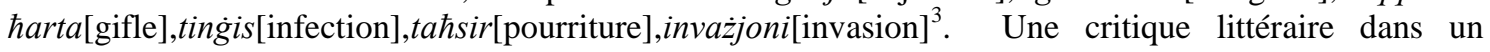
magazine scolaire du conte FirdaminnKelb[Séparation d'un chien] de l'écrivain Ellul Mercer, qui figure au programme du maltais pour les collégiens, affirme que l'auteur utilise le maltais safi [pur] parce que sémitique ${ }^{4}$. 
Les «fautes » ou aberrations dénoncées par ces personnages concernent le plus souvent le lexique, au niveau des mots individuels et de tournures diverses, et moins fréquemment des formes morphosyntaxiques. On visera à ce point les divers niveaux d'analyse linguistique auxquels la variation se manifeste.

\section{Le matériau variationnel en maltais : lexique, syntaxe, morphologie et phonologie}

La variation morphologique existe en maltais et pourrait par exemple être étudiée du point de vue des formes verbales utilisées avec une plus grande fréquence dans les contextes formels et informels. Quant à la variation phonologique, on la remarque tant chez les locuteurs qui parlent des dialectes ruraux, non standard, stigmatisés, que chez les locuteurs du dialecte affecté du maltais, qui semble en quelque sorte empreint de l'influence de la prononciation de l'anglais. L'analyse d'un corpus oral (100,000+ mots) et écrit $(50,000+)$ du maltais $(X X X, 201 X)$ révèle que la variation touche d'autres niveaux de description de la langue. Le corpus oral a été construit à partir d'interactions naturelles dans lesquelles étaient impliqués un nombre réduit $(<10)$ de locuteurs maltais qui se sont laissé enregistrer dans des situations marquées par différents degrés de formalité, la plupart du temps sans être conscients de la présence de l'appareil d'enregistrement. La partie du corpus écrit correspondant à l'écrit informel consiste dans du clavardage (8,144mots). Trois types de textes constituent le corpus écrit formel : de la prose littéraire contemporaine, des écrits administratifs et des articles divers. Ce corpus montre que le niveau lexical est bien intéressé par la variation stylistique ; il s'en dégage par exemple une série d'adverbes et de conjonctions (comme $i z d a$ [mais], minkejja + nom[en dépit de + nom], (fil)waqt li[alors/pendant que], madan(a)kollu[tout de même] XXX 201X : 255) virtuellement réservés à des emplois dans un cadre formel, notamment à l'écrit, et, à l'autre bout de l'échelle, du lexique utilisé uniquement dans des situations marquées par des relations de familiarité (sormi[mon cul], partita [plein de], kif inћlibt [comme je me suis crevée], termes relevés dans l'interaction verbale de l'Infirmière, XXX 201X : 274-5).

La même analyse illustre la variation syntaxique selon le degré de formalité des situations de communication, avec un décalage net entre schémas de jonction propositionnelle. L'étude de la complexité syntaxique dans les contributions d'un locuteur professionnel de la parole publique (un Député parlementaire) montre que la subordination n'est pas du tout rare à l'oral, même dans la conversation spontanée en situation de familiarité, mais le degré d'intégration de subordonnées est beaucoup plus important dans les textes produits dans des contextes institutionnellement formels ou médiatiques. Ainsi, dans le texte informel En famille, le troisième degré de dépendance propositionnelle est atteint seulement deux fois ; dans tous les autres cas de subordination, on ne trouve que le premier, et, plus rarement, le deuxième degrés (ex. 1):

\begin{tabular}{|l|l|}
\hline (1) L1: $\left[{ }^{\mathrm{M}=\text { Matrice }}\left[{ }^{1}\right.\right.$ meta ha tispic̀ća l-iskola $\left.{ }^{1}\right]$ ha & $\begin{array}{l}\mathrm{L} 1:\left[{ }^{\mathrm{M}}\left[{ }^{1} \text { quand tu finis l'école }{ }^{1}\right] \text { tu commenceras }\right. \\
\left.\text { tibda }\left[{ }^{2} \text { taghmel din }{ }^{2}\right]^{\mathrm{M}}\right] \text { ? }\end{array}$ \\
DEPUTE : EN FAMILLE & \\
\hline
\end{tabular}

Par contre, dans le texte formel Parlement, les totaux atteignant environ dix séquences verbales subordonnées dans les énoncés ne sont pas rares. Dans l'exemple 2, on remarque un total de onze séquences verbales subordonnées, même si l'énoncé atteint seulement le huitième degré d'intégration, à cause de la redondance dans la structuration syntaxique, qui relève d'une technique de persuasion usuelle de la rhétorique politique :

(2) L2: aћna m'ghandniex nibżgћu / inbiddlu ghat-tajjeb / u [ ${ }^{\mathrm{M}}$ ghandna $\left[{ }^{1} \mathrm{jkollna}\right.$ quddiem / ghajnejna / lil $\mathrm{da}=\mathrm{k}$ kollha / $\left[{ }^{2}\right.$ li jkunu $\left[{ }^{3}\right.$ qeghdin $\left.\left.\left[{ }^{4} \text { ibatu }\right]^{3}\right]^{2}\right]$ / $\left[{ }^{5}\right.$ biex nirrangaw is-sitwazzjoni / $\left[{ }^{6}\right.$ halli hajjithom ukoll / tkun [ ${ }^{7}$ tista' $\left[{ }^{8}\right.$ tmur ghall-
L2: nous ne devons pas avoir peur / de changer pour le mieux / et $\left[{ }^{\mathrm{M}}\right.$ nous devons $\left[{ }^{1}\right.$ garder à l'esprit / tous ceux $\left[{ }^{2}\right.$ qui sont $\left[\left[{ }^{3+4} \mathrm{en}\right.\right.$ train de souffrir $\left.\left.\left.^{4+3}\right]\right]^{2}\right]$ / $\left[{ }^{5}\right.$ pour améliorer la situation / $\left[{ }^{6}\right.$ pour que leur vie aussi / puisse 
ahjar $\left.\left.\left.\left.{ }^{8}\right]^{7}\right]^{6}\right]^{5}\right] /\left[{ }^{6}\right.$ halli huma wkoll / jibdew [ ${ }^{7}$ jghixu ahjar $\left.\left.^{7}\right]^{6}\right]$ / [ ${ }^{6}$ halli ntejbu l-livell tal-hajja ta' dawn in-nies $\left.]^{6}\right]^{1}$ ]

DEPUTE : PARLEMENT $\left.\left.\left[\left[^{7+8} \text { s'améliorer }^{8+7}\right]\right]^{6}\right]^{5}\right] /\left[{ }^{6}\right.$ pour que eux aussi / commencent $\left.\left[{ }^{7} \text { à vivre mieux }{ }^{7}\right]^{6}\right] /\left[{ }^{6}\right.$ pour améliorer le niveau de vie de ces gens $\left.\left.{ }^{6}\right]^{1}\right]^{\mathrm{M}}$ ]

Les chiffres correspondent, dans ces cas d'oral institutionnellement formel,à ceux obtenus dans un texte écrit formel (ex. 3). Entre le texte écrit pour un journal et les textes oraux formels, les différences majeures résident en effet plutôt dans les libertés ou les contraintes face aux règles syntaxiques, que dans le degré de dépendance des séquences :

(3) $\left[{ }^{\mathrm{M}} \mathrm{Ma}\right.$ jaghmilx iktar sens [ ${ }^{\mathrm{I}}$ li tinghatalhom ilmedicina, [ ${ }^{2}$ halli jkunu [ ${ }^{3}$ jistghu [ ${ }^{4}$ jibqghu fi djarhom u fil-komunita' [ ${ }^{5}$ fejn ikunu [ ${ }^{6}$ twieldu, trabbew u ghexu ${ }^{6}$ [ [ ${ }^{7}$ ghal kemm jista' [ ${ }^{8}$ jkun żmien possibbli $\left.\left.{ }^{8}\right]^{7}\right],\left[{ }^{9}\right.$ fejn ikunu fost in-nies $\left[{ }^{10} \mathrm{li}\right.$ tant ihobbu u li jhobbuhom $\left.\left.\left.\left.\left.\left.\left.\left.{ }^{10}\right]^{9}\right]^{5}\right]^{4}\right]^{3}\right]^{2}\right]^{1}\right]^{\mathrm{M}}\right]$ ?

DEPUTE : ARTICLE

\begin{abstract}
$\left[{ }^{\mathrm{M}}\right.$ Est-ce que cela n'a pas plus de sens [ ${ }^{1}$ qu'il leur soit donné les médicaments, $\left[{ }^{2+3}\right.$ pour qu'ils puissent $\left[{ }^{4}\right.$ rester chez eux et dans la communauté $\left[\left[^{5+6}\right.\right.$ où ils sont nés, où ils ont été élevés et ont vécu $\left.\left.{ }^{6}\right]\right]\left[\left[^{7+8}\right.\right.$ aussi longtemps que possible $\left.\left.{ }^{8+7}\right]\right]$, [ ${ }^{9}$ où ils sont parmi les gens [ ${ }^{10}$ qu'ils aiment tant et qui les aiment? $\left.\left.\left.\left.\left.\left.\left.{ }^{10}\right]^{9}\right]^{5}\right]^{4}\right]^{3}\right]^{2}\right]^{1}\right]^{\mathrm{M}}$ ]
\end{abstract}

Le fonctionnement de la dislocation contribue lui aussi à illustrer la variation stylistique au niveau syntaxique. La dislocation, ou détachement d'un segment de l'énoncé à gauche ou à droite de la partie centrale de l'énoncé, souvent avec la repriseanaphorique ou cataphorique du segment détaché à l'intérieur de la partie centrale, apparaît, en maltais, comme un phénomène essentiellement caractéristique de l'oral. Plus le contexte de la communication est formel, plus l'écart devient important entre, d'une part, la dislocation à contenu lexical, où le segment disloqué peut avoir une structure plus ou moins complexe (ex. 4), et, d'autre part, la dislocation à pronom (ex.5) ou à démonstratif (ex. 6), ces deux types devenant plus fréquents dans les situations où la distance entre interlocuteurs est moins importante et où le sujet est moins sérieux :

\begin{tabular}{|l|l|}
\hline (4) ghandkomkulldritt li ma ttuniex il-*pairing* / & vous avez tous les droits de ne pas nous donner le \\
bi KSUR tal-prattika / li ssirf'diversipajjiziohrajn & pairage / par violation de la pratique / qui se fait \\
/ ghandkomdritt / immambaghadghandidritt li & dans divers autres pays / vous avez le droit / mais \\
jiena / id-drittijiettal-maġgoranza li niggverna / & après j'ai le droit que moi / les droits de la \\
u li niggverna u nkunpreżenti / fil-fora & majorité que je gouverne / et que je gouverne et \\
nternazzjonali / nissalvagwardjah & que je sois présent / dans les forums \\
VICE PREMIER MINISTRE : PARLEMENT & internationaux / jele sauvegarde \\
\hline
\end{tabular}

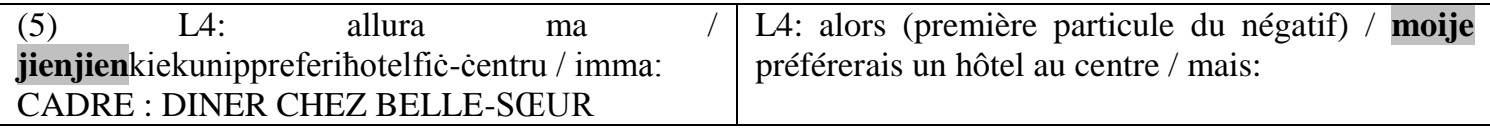

(6) L4: da= // tant qaghadbil-ghaqal / li l*hostess* haditu fil-kabina< à propos de son fils> CADRE : DINER CHEZ BELLE-SEER
L4: celui-ci // il s'est tenu tellement tranquille / que l'hôtesse l'a emmené dans la cabine 


\subsection{Le matériau variationnel en français: lexique, syntaxe, morphologie et phonologie}

Si en France, comme on a déjà vu, la variation phonologique de type régional est mitigée, le niveau du phonique occupe par contre une place importante parmi les phénomènes socialement et situationnellement variables en français : "Les phénomènes variables les plus saillants relèvent du phonique (surtout prosodie) et du lexical, et dans une moindre mesure de la morphologie et de la syntaxe. » (Gadet 2007 : 63).

Sur le plan segmental, on trouve, entre autres traits, les simplifications des groupes consonantiques, comme dans chais pas ou chpas pour je (ne) sais pasou $i$ 'savent pas pour ils (ne) savent pas; l'élision de certaines voyelles comme dans t'as voulu et l'apparition du $e$ instable à des endroits où selon la norme il devrait être interdit, comme en finale (mêmeu au lieu de même). La liaison est définie comme « un phénomène privilégié » de la variation (Gadet 2007: 65), et permet d'opposer les fausses liaisons réalisées par hypercorrection, très recherchées, à la rareté de la liaison chez certains locuteurs / à certaines occasions. Sur le plan supra-segmental, les phénomènes variables concernent la place de l'accent dans le groupe rythmique, le débit, et la courbe intonative (2007: 64). Au niveau morphologique, on peut mentionner, l'emploi ou l'évitement de certains temps verbaux, la fréquence des temps verbaux périphrastiques, et la substitution ou modification des pronoms (par exemple, ils pour elles ; BlancheBenveniste 1997, Gadet 1997 et 2007). La variation syntaxique implique la réalisation des structures interrogatives (Gadet 1997), les modalités de jonction dans les phrases complexes, et les relatives non standard, comme dans les gens qu'on les remet pas à leur place / ils se prennent des ailes et la cheminée que j'aime y faire du feu (exemples empruntés à Gadet 1997, voir aussi Gadet 1995), ou bien le patron que je travaillais pour m'est rentré dedans question pognon ${ }^{5}$.

Au sein du matériau variationnel en France, le lexique ressort à cause du vocabulaire parallèle. En fait, George (1993) démontre que pour certaines notions, la variation lexicale va bien au-delà du simple synonyme unique. Dans ces cas le nombre de synonymes informels, non standard, l'emporte de loin sur celui des synonymes formels et neutres. George fournit pour le verbe partir deux alternatives relevant du strictement formel (se retirer, prendre congé), six termes neutres (partir, s'en aller, s'échapper, s'enfuir, s'évader, s'éloigner) et non moins de trente-trois alternatives relevant de l'informel (filer, se casser, se débiner, s'arracher, se dévisser, foutre le camp, débarrasser le plancher, tricoter des pincettes, prendre la tangente ...), liste qu'il dit non exhaustive (George 1993 : 157-8). Pour bien des notions, il s'agit donc de distribution tripartite, selon l'emploi dans des situations formelles, l'emploi neutre, et l'emploi dans des situations de familiarité ou de solidarité au sein d'un groupe où la question de l'appartenance se règle aussi à travers le lexique utilisé.Les exemples suivants illustrent cette variation lexicale selon le degré de formalité :

- Je me restaurais quand entra dans ma vie celle pour qui je brûle d'une passion folle.

- J'étais en train de manger quand j'ai rencontré celle que j'aime tant.

- J'cassais la dalle quand j'l'ai vue, la nana que j'ai dans la peau. ${ }^{6}$

Pour faire le bilan de cette comparaison du matériau variationnel dans les deux langues, nous pouvons dire que ce matériau se présente à tous les niveaux d'analyse linguistique, mais à des degrés de saillance particuliers pour tel ou tel niveau dans chaque langue : faible variation régionale en français versus forte variation diatopique en maltais, saillance du lexique et du phonétique social / stylistique en français... Des études ultérieures sont nécessaires pour que l'on puisse pourtant affirmer avec certitude à quels niveaux la variation est particulièrement saillante en maltais. Ce qui est sûr, c'est que la forte variation par alternance codique entre deux langues différentes, virtuellement omniprésente dans le cadre maltais, ne figure pas dans les mêmes proportions dans le cadre français, car en France, l'alternance codique principale (français-arabe), ne touche qu'une partie de la population, et est utilisée pour des motivations autres que l'alternance maltais-anglais à Malte. La question des motivations est pourtant étroitement liée à l'évaluation, et nous amène à une discussion sur les attitudes vis-à-vis de ces divers types de variation. 


\subsection{L'acceptabilité sociale de la variation aux différents niveaux d'analyse linguistique}

Nous ferons l'hypothèse que des niveaux de variation mentionnés ci-dessus pour le maltais, c'est la variation morpho-syntaxique qui est surtout tolérée. On entend souvent, même à la télévision, des locuteurs qui transgressent les règles d'accord entre noms/pronoms et adjectifs ou entre sujets et verbes, ce qui passe (presque) inaperçu à l'oral. On note aussi l'emploi fréquent d'adjectifs au lieu d'adverbes

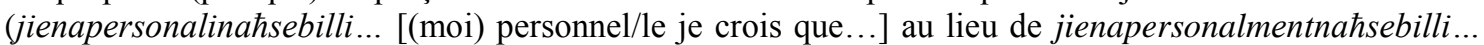
[(moi) personnellement je crois que...]. On entend de fréquentes transgressions aux règles de la relative, avec des emplois non standard concernant les conjonctions illi/li [que] et fejn[où]. Par contre, il semble qu'au niveau lexical, de nombreux termes choquent et sont sujets à des jugements dépréciatifs de la part d'une section de la population ${ }^{7}$. Même des termes traditionnellement considérés comme standard comme

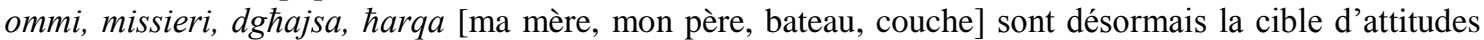
négatives en tant que termes déclassants, à cause de leur sonorité sémitique (réelle ou supposée). De même, au niveau du phonique, si les non-dialectophones peuvent déclarer des attitudes approbatrices de l'existence des dialectes, en avançant des arguments de pittoresque, de pureté, d'ancienneté, de patrimoine, la plupart font ensuite paradoxalement preuve d'attitudes condamnatrices face à leur utilisation, notamment hors de la sphère de la familiarité ou des groupes de solidarité, en s'appuyant sur des arguments d'ignorance / de criardise ${ }^{8}$.

Quant à l'acceptabilité sociale de la variation aux différents niveaux en français, il semble que la variation lexicale soit «admise» (Gadet $1997: 114)$. Ceci n'exclut pas des limites situationnelles : la recherche de Armstrong (2001 : 143) montre des attitudes normatives de la part des participants à son corpus de Dieuze face à l'emploi d'items lexicaux non standard.

Armstrong (2001) élabore une thèse intéressante quant à la tolérance sociale vis-à-vis de la variation phonique et grammaticale en France. Il remet en question la théorie avancée par Hudson quant à la suppression de la variation grammaticale et à l'épanouissement de la variation phonologique, supposés caractériser toutes les communautés :

$[D]$ ifferences in syntax tend to be suppressed, whereas those in pronunciation and vocabulary tend to be favoured and used as markers of social differences. There do not appear to be any examples of communities in which this relationship is reversed, with less variation in vocabulary and pronunciation than in syntax. (Hudson 1996: 45).

Pour Armstrong, ce qui est à l'origine de la distinction de la situation française face à cette tendance, c'est la phonologie hautement standardisée du français, qui donne lieu à une distribution socio-stylistique particulière des phénomènes variables au niveau de la grammaire. Armstrong considère les tendances observables au Royaume-Uni, pour tirer des conclusions concernant la France. Au Royaume-Uni, les différences de prononciation sont fortes d'une ville/d'une zone à l'autre, mais il existe un schéma net selon lequel certains groupes de locuteurs évitent les formes grammaticales standard alors que d'autres évitent les formes non-standard de manière presque totale (2001: 125) :

[In the UK] standardisation can be argued to exert normative pressures more effectively on the grammatical level. [...] Standard grammatical forms are perceived therefore as being readily available to those who wish to learn them, while phonological forms are not, or less so. » (Armstrong 2001: 127)

En anglais britannique, la grammaire non standard s'en trouve par conséquent plus stigmatisée que la phonologie, car là où les traits phonologiques variables connotent l'origine régionale du locuteur, la grammaire variable est évaluée comme étant plus 'cognitive' ; elle évoque le niveau d'éducation d'un locuteur et son rapport à la littératie. Armstrong (2001) appuie son argument sur la proposition de Romaine $^{9}$ qu'il existe une forte conscience chez les locuteurs de la classe moyenne relativement à la stigmatisation de la morphologie non standard. Pour Armstrong, ce schéma de variation grammaticale en anglais s'organise selon une distribution sociale différente de la variation grammaticale en français. Gadet (1997) démontre, par l'exemple de la fréquence des réalisations des nombreuses structures interrogatives 
possibles en français, que dans cette langue, s'il y a bien des périphéries de formes prestigieuses ou familières/populaires qui sont exclusivement utilisées (respectivement) par la classe moyenne en situation formelle (dont quand Jeanne vient-elle? et quand venez-vous ?) et par la classe ouvrière (dont quand que vous venez ?; quand c'est que vous venez ?; et quand que c'est que vous venez ?), il existe aussi un noyau de formes non standard qui sont employées par la communauté entière, toutes classes confondues (dont quand viennent les enfants ?; quand est-ce que vous venez ?; vous venez quand ?; etquand vous venez ?).

Les locuteurs français partagent ainsi des normes de comportement et d'évaluation au niveau de la grammaire variable qui diffèrent du schéma d'emploi et de stigmatisation présent pour l'anglais britannique : les locuteurs français tolèrent, voire favorisent la variation au sein du noyau de variation syntaxique puisqu'elle constitue une des ressources majeures qui leur permettent d'exprimer leur identité sociale (Armstrong 2001). La variation grammaticale en vient donc à compenser la ressource assez limitée pour les locuteurs français de la variation au niveau de la prononciation, allant ainsi à l'encontre de la tendance suggérée par Hudson.

Les Français parlent de l'accent de banlieue, très stigmatisé, ce qui constitue un jugement de classe, plutôt qu'un jugement en fonction de l'élément régional (Ibid) : «La stigmatisation diastratique est encore plus forte que la marginalisation de l'expression diatopique (région ou ruralité). » (Gadet 2007 : 114). La stigmatisation dépasse le comportement linguistique et vise la catégorie sociale elle-même, avec son style de vie, son éducation non poussée, et son rapport réduit à la littératie.

La conscience d'une variété diastratique ne semble cependant pas être forte en contexte maltais, et c'est probablement ce qui explique que l'on mette les deux catégories des locuteurs dialectophones et les locuteurs de variétés populaires dans le même sac des ћamalli [criards] / injoranti [ignorants]. Pourtant, si les dialectes régionaux peuvent aussi dans une certaine mesure être formellement valorisés pour les arguments de patrimoine, de pureté et d'ancienneté, la variation qui émane de la classe ouvrière n'a certainement pas la cote.

Le degré d'acceptation sociale est l'un des facteurs qui influent sur la portée des divers types de variation dans des contextes différents. Toute communauté, avec son histoire, ses pratiques et ses configurations diverses, accorde plus ou moins d'importance à la variation stylistique, à partir de la place qu'occupent les autres types de variation dans le groupe. Ceci a permis que soit esquissée une typologie des langues selon cet aspect, dont nous allons brièvement rendre compte pour ensuite nous focaliser sur les cas français et maltais.

\section{Hypothèses sur les types de variation proéminents dans des langues spécifiques}

Berruto (1987) constate, pour la situation italienne, la prédominance de la variation diatopique, universellement présente pour tous les locuteurs, car en Italie, le standard n'existe guère sans élément régional : des variétés régionales standard constitueraient la norme sociale dans chaque région. Berruto parle de «l'italien néo-standard», sensible à la différentiation diatopique, correspondant dans l'usage concret à un italien régional moyen des classes avec un certain niveau d'éducation. Le locuteur éduqué révèle d'habitude ses origines au moins par sa prononciation et par l'intonation, même s'il n'a pas d'accent régional très évident.

La différentiation sociale viendrait en deuxième rang, avec une distinction entre l'italien régional des classes au niveau d'éducation poussé et l'italien régional populaire (Ibid.). La variation diaphasique occupe le troisième rang : chaque variété socio-géographique a en principe ses propres formes de variation diaphasique (Ibid.).

Cette hiérarchie diffère de celle du Royaume-Uni, où la variation diastratique domine les usages. Selon Wells (1982), au Royaume-Uni, à partir du lexique et de la prononciation utilisés, les personnes peuvent tirer des jugements immédiats sur l'appartenance d'un inconnu à telle ou telle classe sociale. Pour le cas 
du Royaume-Uni, la relation entre l'accent et le statut social peut être représentée par le symbole de la pyramide comme dans le schéma 1.

RP (ReceivedPronunciation)

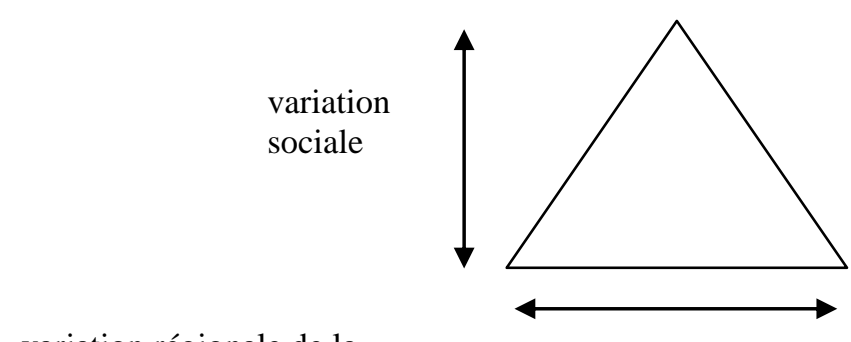

variation régionale de la

prononciation

SCHEMA 1 : Le modèle triangulaire de la relation entre statut et accent (source : Hughes A., Trudgill P., Watt D. $2005: 10)$

Les locuteurs de la classe ouvrière font preuve de beaucoup de variété régionale, mais les locuteurs de la classe dominante, locuteurs de la variété ReceivedPronunciation, ne signalent pas leur origine régionale dans leur discours. L'indication de la provenance régionale se trouve par la suite d'amplitude plus réduite que l'indication de l'appartenance sociale. La variation diastratique se voit accorder tellement beaucoup d'importance que les attitudes linguistiques vont au-delà des jugements sur les variétés elles-mêmes pour viser jusqu'aux compétences cognitives des locuteurs : les locuteurs des variétés de prestige sont évalués comme possédant des traits de statut comme l'intelligence, le succès et la confiance en soi ${ }^{10}$.

\subsection{Le cas de la France}

Pour la situation de la France, Gadet $(1998,2007)$ postule la proéminence du diaphasique, en basant son hypothèse également sur les arguments avancés par Sanders (1993) : l'importance de l'écart entre le français parlé et écrit, comme de la distinction entre emplois formel et informel, marquent des différences majeures entre les situations française et britannique, et le registre apparaît comme phénomène de grande ampleur en français.

Gadet (1998) retrace la progression du diaphasique au fil du temps. Le français aurait connu une période au XIXe siècle, où dominait la variation diatopique, au-delà du français, car la plupart des gens parlaient les langues régionales et n'avaient aucune connaissance du français, ou n'en avaient qu'une connaissance passive. Ceci fut suivi par une période de prédominance diastratique, lors du processus d'industrialisation et d'urbanisation, «époque de fortes distorsions entre «langues de classes », qui correspond aussi à la francisation radicale » (1998: 62). De nos jours, sous l'effet de la standardisation et du nivellement, la diversité diatopique n'est plus saillante en France. La différentiation diastratique s'est aussi réduite : d'une part, avec la démocratisation du français standard, grâce à l'éducation universelle et à la prolongation de la scolarité, et d'autre part avec l'acceptation et la généralisation de formes (notamment lexicales) de l'argot ou populaires dans le discours utilisé par toute sorte de locuteurs (Gadet 2003) dans les situations informelles ${ }^{11}$. Des mots soigneusement évités par l'élite et les classes moyennes au début du XXe siècle font maintenant partie intégrante du répertoire linguistique de tous (Gadet 1998, 2007). C'est ainsi la variation diaphasique qui occupe le primat sur les autres types de variation. 


\subsection{Le cadre maltais}

Le cas maltais se complique par la présence sur un territoire minuscule de tendances et de langues et variétés multiples. Nous tenterons d'émettre une hypothèse quant à la primauté d'un type de variation à partir d'une réflexion basée sur des constatations de nature générale.

Il nous semble qu'à Malte comme en Grande-Bretagne, les considérations de statut social dominent les esprits. De nombreux parents s'efforcent à ce que leur communication avec leurs enfants soit en anglais, ou en maltais «standard » mais de préférence intégrant de fréquents éléments anglais. C'est aussi sous le poids des pressions sociales que les adultes pratiquent l'alternance codique pour affirmer leur statut (professionnel, public ou social).

L'influence du statut social serait d'ailleurs à l'origine de la régression de la variation diatopique. Les parents dialectophones soucieux de pousser l'ascension sociale de leurs enfants s'assurent que ceux-ci adoptent le maltais standard, voire peut-être l'anglais comme L1. Sous l'effet de l'urbanisation, les anciens villages devenus zones résidentielles (et souvent commerciales) majeures, témoignent du nivellement en faveur du standard. Pour la vaste majorité des dialectophones, la vie sociale se divise en deux schémas distincts, dont les bornes sont aussi linguistiquement définies : d'une part, il y a l'emploi du dialecte régional avec les membres de la communauté, par solidarité, et d'autre part, il y a changement vers le standard en présence d'inconnus, et dans des situations plus ou moins formelles.

Une différence importante avec le modèle anglais réside dans le fait qu'en Grande-Bretagne, il n'y a qu'un pourcentage assez réduit de locuteurs de la variété ReceivedPronunciation, dépourvue d'indices de provenance régionale. A Malte, le pourcentage de locuteurs du «standard», ou de non-dialectophones, doit être estimé comme bien plus important.

A notre avis, la variation diaphasique est aussi très importante. Les données constatées dans le corpus de XX (201X) attestent les comportements linguistiques extrêmement variables des locuteurs, qui peuvent être amenés, selon les situations dans lesquelles ils se trouvent, à passer du maltais le plus informel, spontané et relâché (au niveau du lexique et de la morpho-syntaxe), à un maltais très formel, parfois «calculé », ou bien à l'alternance codique ou encore à l'emploi de l'anglais dans des situations d'accommodation ou lorsqu'ils veulent projeter telle ou telle identité sociale ou endosser tel ou tel rôle public ou professionnel. Deux cas sont retenus ici pour illustrer ce va-et-vient entre styles. L'Assistante pharmacienne, mariée à un professeur de l'université, tend à intégrer l'anglais à divers degrés dans ses propos selon les situations dans lesquelles elle se trouve. Ses interactions avec sa famille se caractérisent normalement par le maltais langue dominante, parfois relâché (ex. 7), mais elles peuvent contenir des termes ou expressions anglais, lorsqu'elle parle de thèmes qui affirment son statut bourgeois, comme la bonne (ex. 8), ou les études de son fils (ex. 9) :

\begin{tabular}{|c|c|}
\hline $\begin{array}{l}\text { (7) L3: LE MHUX HEMM < L1, son neveu, a } \\
\text { remis dans son assiette une bouchée de nourriture } \\
\text { qu'il a trouvée trop chaude ; rires > / } \\
\text { XXXXXXXX } \\
\text { [...] } \\
\text { L3: kiekunghidlu KULHA / itfaghhagohalqekejja } \\
\text { ASSISTANTE PHARMACIENNE : DINER }\end{array}$ & $\begin{array}{l}\text { L3: non pas là < rires }>/ \mathrm{XXXXXXXX} \\
{[\ldots]} \\
\text { L3: moi je lui dirais mange-la / mets-la dans ta } \\
\text { bouche allez }\end{array}$ \\
\hline
\end{tabular}

(8) L2: (kellek il-*maid* illum?)

L3: is-soltunghidilhatigi $*$ Tuesday* / imm= aћna m'ahniexdaqshekk *fixed* ta / naghtu kas nghidilha / gielitghid / tghidlitista' *Monday* jew *Wednesday* inti ?

ASSISTANTE PHARMACIENNE : DINER
L2: tu avais la *maid* (bonne) aujourd'hui ? L3: d'habitude je lui dis de venir le *Tuesday* (mardi) / mais nous la journée c'est pas tellement $*$ fixed $*$ (fixe) tu sais / par exemple je lui dis / parfois elle dit / elle me dit tu peux *Monday* (lundi) ou *Wednesday* (mercredi) tu crois? 
(9) L3: qallekkemm mar tajjeb (*French*?)? L5: mhm L3: *I think it's his best subject* ASSISTANTE PHARMACIENNE: DINER

L3: il t'a dit qu'il a très bien réussi son
*French* (français)?
L5: mhm
L3: *I thinkit'shis best subject* (je crois que
c'est sa meilleure matière)

Dans des situations plus formelles, l'anglais est susceptible de devenir la langue dominante dans ses propos. Pour Romaine (1995), l'emploi de la langue extra-communautaire peut être vu comme l'expression linguistique du rejet d'un type de vie. Dans le cas de cette locutrice maltaise, le choix de l'anglais pourrait signifier le rejet du style de vie et de l'identité insulaire, l'affirmation d'une distance voulue entre soi-même et les personnes communes, à mentalité restreinte, et parallèlement, il s'agirait d'une affirmation qu'elle embrasse une mentalité plus ouverte, moderne, européenne. L'alternance est donc chez cette locutrice métaphorique ; elle connote cette affirmation d'appartenance à un groupe et la stigmatisation d'un autre. Sa transaction dans une compagnie financière reflète cette signification de l'alternance. L'anglais, marqué par une forte alternance avec le maltais, semble être la L1 de l'agent financier (L1), copropriétaire de l'agence, qui la reçoit. L'Assistante pharmacienne (L2) tente de se montrer à la hauteur de la situation en émulant cette variété :

\begin{tabular}{|c|c|}
\hline 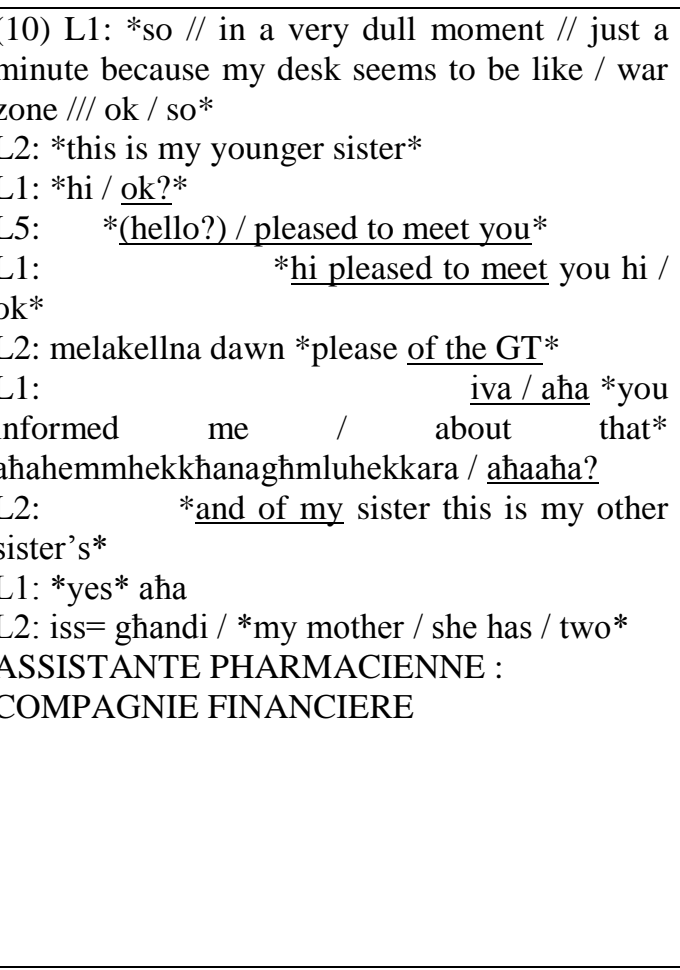 & $\begin{array}{l}\text { becausemy desk seems to belike / war zone /// ok } \\
\text { / so* (alors // en un moment vraiment glauque // } \\
\text { une minute seulement parce que mon bureau est } \\
\text { comme / une zone de guerre /// donc / alors) } \\
\text { L2: *this is my younger sister* (elle, c'est ma } \\
\text { soeurcadette) } \\
\text { L1: *hi / ok?* (bonjour / ça va ?) } \\
\text { L5: *(hello?) / pleased to meet you* (bonjour } \\
\text { / enchantée) } \\
\text { L1: } \\
\text { (bonjour / enchanté / bonjour / ok) } \\
\text { L2: alors nous avions ceux-ci *pleaseof the GT* } \\
\text { (s'il vous plaît de la GT) } \\
\text { L1: } \\
\text { d'accord*youinformed me / about that* ( vous } \\
\text { m'avez informé à propos de cela) oui là nous } \\
\text { allons faire comme ça tenez / oui oui? } \\
\text { L2: } \\
\text { *and of mysisterthisismyothersister's* (et de ma } \\
\text { soeur celui-ci est de mon autre soeur) } \\
\text { L1: *yes* (oui) oui } \\
\text { L2: et puis j'ai / *mymother / she has / two* (ma } \\
\text { mère / elle en a deux) }\end{array}$ \\
\hline
\end{tabular}

Dans des situations de formalité institutionnelle, protocolaire, la formalité n'est pas marquée par le recours à l'anglais, mais plutôt par son évitement, au profit de l'emploi du maltais recherché, «calculé », mentionné ci-dessus. Ce genre de maltais apparaît chez les locuteurs professionnels de la parole publique, comme dans l'exemple 4 ci-dessus, et abonde en emplois de termes spécialisés, et, syntaxiquement, en occurrences de la voix passive, de relatives, complétives et autres propositions subordonnées, parmi d'autres structures plus ou moins complexes. 
Ces emplois de variétés spécifiques vont dans le sens de confirmer la constatation de Coupland (2001), se basant sur Ochs (1988), à propos des situations formelles : les communautés ont souvent des variantes de prestige distinctes pour différentes activités. Les données de Ochs sur l'île Samoa relèvent la distinction entre variantes de prestige particulières pour des activités liées au monde occidental, comme l'éducation formelle, le commerce urbain et les religions occidentales, et d'autres variantes de prestige pour les activités traditionnelles comme les rencontres formelles, les rites de passage et autres cérémonies. A Malte, on trouve une répartition similaire entre les domaines de la formalité sociale, correspondant à l'émploi de toute une gamme d'anglais, (communications et documents écrits d'entreprises, colloques, communication orale d'une partie de l'élite sociale ou de personnes aspirant à afficher leur statut social), et les domaines de la formalité institutionnelle ou protocolaire, correspondant à l'emploi du maltais formel (Parlement, rassemblements de masse, tribunaux, cérémonies officielles, église).

\section{Conclusion}

Au terme de cette discussion sur les convergences et les divergences dans les schémas de variation en contexte maltais et français, nous constatons que les situations sociolinguistiques présentes dans les deux pays diffèrent sensiblement sur plusieurs points, les causes principales étant les cadres bilingue à Malte et relativement monolingue en France. A cette réalité se joignent des facteurs socio-historiques, comme le très vieux règne absolu de la norme en France, qui a eu pour conséquence à l'autre extrême de la palette linguistique le foisonnement de formes familières, non standard, divergeant d'une manière particulièrement saillante du standard normé. Le processus de standardisation en maltais est beaucoup plus jeune, et se focalise notamment sur l'orthographe. La politique linguistique dans les deux pays doit gérer l'intrusion de l'anglais, mais un écart énorme sépare dans les deux contextes les proportions et l'agressivité des méthodes d'intrusion de cette langue, tout comme les enjeux qu'elle représente. La question des attitudes vis-à-vis de cette intrusion et de la variation en général admet également de nombreuses colorations diverses d'un pays à l'autre. 


\section{Références Bibliographiques}

\section{XX (201X)}

Armstrong, N. (2001). Social and Stylistic Variation in Spoken French: A Comparative Approach. Amsterdam: John Benjamins.

Barbara, V. V. (1990). Ilsienna u l-bandierataghna. Blacktown, NSW - Australie: V. V. Barbara.

Bell, A. (2001). Back in style: Reworking audience design. In P. Eckert, J.R. Rickford (éds.), Style and sociolinguistic variation (139-169). Cambridge: CUP.

Berruto, G. (1987). Sociolinguistica dell'italiano contemporaneo. Rome: Carocci.

Blanche-Benveniste, C. (1997). Approches de la langue parlée en français. Paris: Gap.

Blom J., Gumperz J. (1972). Social meaning in linguistic structure: Code-switching in Norway. In J. Gumperz, D. Hymes (éds.), Directions in sociolinguistics (407-434). New York: Holt, Rinehart and Winston Inc.

Borg, A., Mifsud, M. (2005). Il-pożizzjoni tal-Malti f’Malta.Il-Malti 77, 6-16.

Caruana, S. (2011). Bilingualism and language policy in Malta. In P. Ramat, E. Miola (éds.), Language Contact and Language Decay: Socio-Political and Linguistic Perspectives (11-46). Pavia: IUSS.

Cassar, C. (2001). Malta: Language, literacy and identity in a Mediterranean island society. National Identities 3(3), $257-275$.

Catach, N. (1993). The reform of the writing system. In C. Sanders (éd.), French Today: Language in its Social Context (139-154). Cambridge: CUP.

Coupland, N. (2001). Language, situation, and the relational self: theorizing dialect-style in sociolinguistics. In P. Eckert, J.R. Rickford(éds.), Style and Sociolinguistic Variation(185-210). Cambridge: CUP.

Coveney, A. (1996). Variability in spoken French: A sociolinguistic study of interrogation and negation. Bristol: Elm Bank.

Diacono, Ġ. (1977). Gheltijiet u barbariżmi fil-Malti. La Valette: Pubblikazzjonijiet Diacono.

Ebejer, F. (1989). The Bilingual Writer as Janus. Malte: Foundation for International Studies.

Gadet, F. (1995).Les relatives non standard en français parlé : le système et l'usage.Etudes romanes de l'Université de Copenhague 34, 141-162.

Gadet, F. (1997). La variation, plus qu'une écume. Langue française 115, 5-18.

Gadet, F. (1998). Cette dimension de variation que l'on ne sait nommer. Sociolinguistica 12, 53-71.

Gadet, F. (2003). La variation : le français dans l'espace social, régional et international. In M. Yaguello (éd.), Le Grand Livre de la langue française (91- 151). Paris :Seuil.

Gadet, F. (2005). Research on Sociolinguistic Style. In U. Ammon et al. (éds.), Sociolinguistics / Soziolinguistik(1353-1360). Berlin et New York : Mouton de Gruyter.

Gadet, F. (2007). La variation sociale en français.Paris :Ophrys.

George, K. (1993). Alternative French. In C. Sanders (éd.), French today: Language in its Social Context (155-170). Cambridge: CUP.

Hudson, A. (1996). Sociolinguistics (2eéd.). Cambridge: CUP.

Hughes, A., Trudgill, P., Watt D. (2005). English accents and dialects: an introduction to social and regional varieties of English in the British Isles (4e éd.). Londres : Hodder Arnold. 
Judge, A. (1993). French: a planned language?. In C. Sanders (éd.), French today: Language in its Social Context (726). Cambridge: CUP.

Lodge, A. (1993). French: From dialect to standard. Londres et New York: Routledge.

Ochs, E. (1988).Culture and Language Development: Language Acquisition and Language Socialization in a Samoan Village. Cambridge: CUP.

Sanders, C. (1993). Sociosituational variation. In C. Sanders (éd.), French today: Language in its Social Context (2753). Cambridge : CUP.

Schilling-Estes, N. (2002). Investigating Stylistic Variation.In J.K. Chambers, P. Trudgill, N. Schilling-Estes (éds.),Language Variation and Change(375-401). Oxford: Blackwell.

Traugott, E., Romaine S. (1985). Some questions for the definition of 'style' in socio-historical linguistics, Folia LinguisticaHistorica VI, 7-39.

\footnotetext{
${ }^{1}$ Le cas de Malte admet aussi un troisième pilier sur lequel se base l'identité nationale : la religion catholique.

${ }^{2}$ L'île a historiquement été un territoire très convoité, par sa position au centre de la Méditerranée. D'où les dominations successives et leurs effets linguistiques. Labase arabe de la langue maltaise, originellement un dialecte de type nord africain, date de la période de domination des îles par les Arabes (870-1090). Sur cette base se sont greffées d'importantes influences romanes (arrivée des Normans en 1090 ; inclusion médiévale de Malte au sein du Royaume de Sicile). Le sicilien joua un rôle considérable dans la formation de la langue. En 1530, Charles Quint donna les îles à l'Ordre des Chevaliers de Saint-Jean, qui les gouverna jusqu'en 1798. Durant cette période, le latin était la langue principalement utilisée pour les documents écrits de l'Ordre, mais la variété toscane, et le sicilien étaient également utilisés. Le pouvoir colonial anglais évinça l'italien de son statut de langue officielle de l'île afin de le supplanter par l'anglais. Ce contact laissa la langue imbibée d'un important élément anglo-saxon.
}

${ }^{3}$ Les trois premiers termes proviennent de Barbara (1990), les trois derniers de Diacono (1977).

${ }^{4}$ Sammut, P. (2014). Kritikaletterarja. FirdaminnKelb. Saghtar 342, 42-43.

${ }^{5}$ Exemple pris du site : w3.gril.univ-tlse2.fr/francopho/lecons/niveaux.html.

${ }^{6}$ Exemples pris du site : leprofesseurdefrancais.blogspot.com.mt/2008/01/exercices-de-lexique-les-registres-de.html.

${ }^{7}$ Ceci vaut naturellement d'autant plus pour les termes et expressions associés aux situations informelles, comme imlejtha l-ћawsla[j'en ai mis plein la panse] utilisés au lieu des termes sociolinguistiquement neutres plus acceptés comme intlejt ou infqajt [j'ai assez/trop mangé].

${ }^{8}$ Ceci est démontré par les résultats d'une enquête relative à ces attitudes linguistiques (XX 201X).

${ }^{9}$ La reference est à Romaine S. 1984, The language of children and adolescents, Oxford, Blackwell.

10 Document British English, disponiblesur http://www.ic.arizona.edu/ 1sp/BritishEnglish.html, citant Giles H. et Sassoon C., 1983, « The effects of speakers' accent, social class background and message style on British listener's social judgments » in LANGUAGE AND COMMUNICATION n 3, pp. 305-313, et Hughes A., Trudgill P. et Watt D. $2005: 11)$.

11 Certains termes passent même au français utilisé dans des situations formelles, voire d'écrit. C'est ainsi qu'on lit à propos de «policiers ripoux » dans la presse, jusque dans les titres principaux. 